\title{
HapRing: A Wearable Haptic Device for 3D Interaction
}

\author{
Oscar Javier Ariza Nunez, Paul Lubos, Frank Steinicke \\ Human-Computer Interaction, Department of Informatics, Universität Hamburg
}

\begin{abstract}
Haptic devices have the capability to offer good solutions in terms of usability and accuracy related to touch feedback on immersive virtual environments (IVEs). However, there are very few affordable devices to perform natural interaction in a $3 \mathrm{D}$ space and some do not represent a suited solution for the common ergonomic and stimuli-meaningfulness issues. In this article, we present a wireless haptic ring (HapRing) for spatial interaction, providing vibro-tactile signals as well as vibration cues on a fingerbasis using a haptic actuator. Other features include inertial measurement, digital input and support for IR camera-based tracking.
\end{abstract}

\section{Introduction}

Providing touch feedback in spatial interactive applications through haptic technology, using devices composed by actuators, makes more meaningful experiences possible by adding sensations as responses to a user's interaction within a VE. Haptic feedback can be dynamically adjusted according to interaction conditions to provide more significant information such as cues and warnings. Accordingly, the creation of versatile and affordable devices to provide haptic feedback for simple spatial interaction tasks has not been addressed completely, leaving room to propose a device for spatial applications in mid-air while keeping the fingertips free to use multi-touch tabletops, enabling active touch feedback in both cases.

Most of the related solutions are focused on multi-modal experiences, centering all the efforts on the senses of sight and hearing and, as a consequence, usually no haptics technology is taken into account. The use of haptic feedback on IVEs would enable the design of new tactile stimuli to effectively convey information in the shape of timely responses, which could be combined with auditory and visual signals, producing better user performance on interactive tasks. Here we present the latest development iteration of HapRing as our proposed device to provide active haptic feedback for spatial interactive applications, eliciting dynamic feedback signals (i.e. vibro-tactile patterns) according to the position between the finger and 3D virtual objects. 


\section{Related Work}

Preliminary research on finger-worn devices explored the creation of input devices as well as some tactile feedback devices (Takafumi et al. 2009; Kouta et al. 2010). Recent work provided real-time auditory and vibro-tactile feedback (Shilkrot et al. 2014; Roumen et al. 2015). Novel techniques in the field, use ultrasonic transducers and air vortex rings to create focused haptic feedback in mid-air (Monnai et al. 2014; Gupta et al. 2013). However, interaction in the 3D mid-air is physically demanding and, therefore, often hinders user satisfaction and performance (Chan et al. 2010). The increase in the DoFs that have to be controlled simultaneously as well as the absence of passive haptic feedback and resulting interpenetration and occlusion issues when "touching the void" (Bruder et al. 2013) are often responsible for reduced performance.

\section{Device Prototyping}
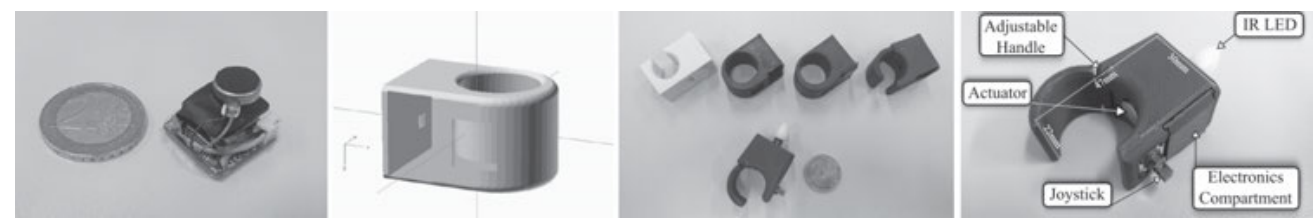

Figure 1: Illustrations of the HapRing design process including the final prototype

HapRing comprises the design and creation of a finger-worn device, avoiding cables by using a low-energy wireless technology. A vibrational element is managed and controlled by embedded software to provide haptic feedback at different frequencies according to signals received from the computer.

\subsection{Hardware and Software}

The embedded software running on the HapRing is managed and controlled by an RF51822 SoC (System on a Chip) based on a 32-bit ARM Cortex, supporting Bluetooth Smart (2.4 GHz Band) for ultra low-power wireless applications. All the components are placed on a small development board $(18.5 \mathrm{~mm} \times 21.0 \mathrm{~mm}$ ) including power connectors (supporting a working voltage from $1.8 \mathrm{~V}$ to $3.3 \mathrm{~V}$ ) and a set of I/O ports used to connect the micro-joystick with command button and the Linear Resonant Actuator (LRA) which is basically a shaft-less vibration motor attached to an active haptic driver (TI DRV2605). As a power source, we used a Lithium Polymer Ion (LyPo) rechargeable battery which outputs a nominal 3.7 V at $40 \mathrm{mAh}$. A voltage converter and micro USB plug were included. An optional Infrared (IR) LED (with $880 \mathrm{~nm}$ wavelength for compatibility with a PPT tracking system) is connected on the ring's top for external reference support. In addition, an IMU is integrated, providing 9-DOF data from a 3-axis combo chip (L3GD20H and LSM303D). For the communication with HapRing, the commands can be sent directly through Bluetooth. We developed an alternative interface application which receives and sends commands through a standard UDP port and transmits them to HapRing. This setup is modular and would allow different computers to communicate with HapRing, as often necessary in laboratories. The HapRing project is intended to be Open 
Hardware, enabling the interested audience to build their own device, based on the 3D printing design files, the embedded software source code, the electronics sketches and the C\# API.

\subsection{Latency and Haptic Feedback}

We measured the latency from the collision detection in the $3 \mathrm{D}$ environment to the vibration signal triggered on the actuator, which was $23 \mathrm{~ms}$, sending a byte-length message to the device using the UDP-like mechanism to send messages with a "no response flag" provided by the Bluetooth LE Specification. According to perception studies (Batteau et al. 2004), the measured latency is sufficiently small based on average hand velocity for common interactive tasks and vibro-tactile related experiments.

To provide haptic feedback in a finger basis, we use a small shaft-less vibration motor (10 $\mathrm{mm}$ diameter, $2.0 \mathrm{~mm}$ height) with an operating voltage of 2.5-3.5 V. We used a PWM driver to control precisely how the motor runs, taking advantage of overdriving and active braking to guarantee responsive and clean signals. According to the vibrator characteristics, the frequencies range is $20 \mathrm{~Hz}$ to $220 \mathrm{~Hz}$. The provided range is sufficient to elicit sensations on the epidermis' tactile corpuscles for haptic feedback related to small displacements and pressure sensations as well as simulation of small texture patterns over movement areas (Cholewiak \& Collins 1991).

\subsection{Manufacturing}

Aiming to create a comfortable, useful and reliable ring device, we iterated over 3D printers, techniques and materials to get a final 3D printed chassis for our device, mount and test the electronics. The ring was sketched, modelled, sliced and $3 \mathrm{D}$ printed according to the constraints imposed by finger ergonomics and electronics. The software tools used were OpenSCAD for 3D modelling, Slic3r for G-Code generation and an Industrial RepRap for the printing labor. Needless to say, the device works just as well for left-handed users as for righthanded users. The finished prototype is illustrated in Figure 1 (right), which shows the various components and the size (47 mm height x $30 \mathrm{~mm}$ width $\mathrm{m} 22 \mathrm{~mm}$ depth).

\section{Testing environment}

To show potential use-cases for HapRing, we extended several applications by providing haptic feedback for interactions, providing an Unity3D plugin to simplify the integration of HapRing. Stereoscopic multi-touch tabletops as well as environments composed by a HMD and a Leap Motion can take advantage of the haptic feedback (Figure 2a and 2b).
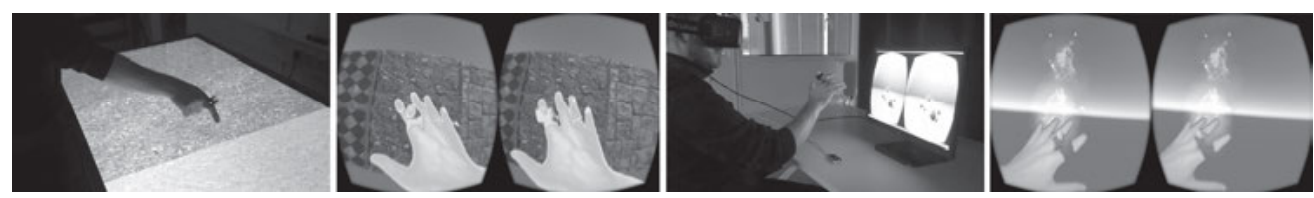

Figure 2. Illustrations of HapRing use cases. 
In both scenarios the user moves their finger while touching a surface and the actuator in the ring is enabled at a certain frequency, depending on the texture touched. Another case (Figure 2c) integrates HapRing into an existing HMD application to pop balloons which appear in the user's area of interaction by touching them. Finally, we developed an interactive application to provide warning cues according to a distance-based vibro-tactile pattern when the virtual hand of the user approaches a fire source (Figure 2d).

\section{Conclusions and future work}

We are currently developing new haptic and interaction techniques including new haptic devices for further research. The presented prototype fulfills the given requirements of a wireless, haptic device, although we still need to evaluate how comfortable it is during long time spans. There are few drawbacks, such as the actuator's vibration being audible in the chassis and minor power leaks requiring more optimizations. Also, we aim to investigate the combination of haptic feedback with acoustic feedback and the effects thereof on user performance within a wide range of setups, for example in VR and AR, as well as tabletops. Other target is the analysis in deep of the vibro-tactile signal patterns and its relationship to the different stages of a hand movement (e.g. ballistic, correction, etc.).

\section{References}

Batteau, L., Liu, A., Maintz, A., Bhasin, Y. \& Bowyer, M. (2004). A study on the perception of haptics in surgical simulation. In Medical Simulation Lecture Notes in Computer Science.

Bruder, G., Steinicke, F., \& Stuerzlinger, W. (2013). Effects of visual con icts on 3D selection task performance in stereoscopic display environments. In Proceedings of ACM 3DUI.

Bruder, G., Steinicke, F., \& Stuerzlinger, W. (2013). Touching the Void Revisited: Analyses of Touch Behavior On and Above Tabletop Surfaces. In Human-Computer Interaction (INTERACT).

Chan, L., Kao, H., Chen, M., Lee, M., Hsu, J. \& Hung, Y. (2010). Touching the void: Direct-touch interaction for intangible displays. In Proceedings of ACM CHI.

Cholewiak, R. \& Collins, A. (1991). Sensory and physiological bases of touch. In Psychology of Touch.

Gupta, S., Morris, D., Patel, S. \& Tan, D. (2013). Airwave: Non-contact haptic feedback using air vortex rings. In Proceedings of ACM Ubicomp. ACM.

Kouta, M., Prattichizzo, D. \& Tachi, S. (2010). Simplified design of haptic display by extending one-point kinesthetic feedback to multipoint tactile feedback. In Haptics Symposium IEEE.

Monnai Y., Hasegawa, K., Fujiwara, M., Yoshino, K., Inoue, S., \& Shinoda, H. (2014). Haptomime: Mid-air haptic interaction with a floating virtual screen. In Procs. of ACM User Interface Software and Technology (UIST).

Shilkrot, R., Huber, J., Liu, C., Maes, P. \& Nanayakkara, S. (2014). Fingerreader: A wearable device to support text reading on the go. In Proceedings of Human Factors in Computing Systems CHI.

Takafumi, A., Hironori, M., Shoichi, H. \& Makoto S. (2009). Haptic ring: touching virtual creatures in mixed reality environments. In Proceedings of SIGGRAPH USA.

Roumen, T., Perrault, S. \& Zhao S. (2015). NotiRing: A Comparative Study of Notification Channels for Wearable Interactive Rings. In Proceedings of Human Factors in Computing Systems (CHI). 